\title{
An Empirical Investigation of Goodness-of-Fit Statistics for Sparse Multinomials*
}

by

\author{
Renneth J. Roehler ${ }^{1}$ and Rinley Larntz ${ }^{2}$
}

Technical Report No. 327

September 1978

* This research was supported in part by Grant NIE-G76-0094 from the National Institute of Education, U.S. Department of Bealth, Education and Welfare.

1 Department of Statistics, Iowa State University

2 School of Statistics, Untversity of Mimesota 
ABSTRACT

Traditional discussions of goodness-of-fit tests for multinomial data consider asymptotic chi-squared properties under the assumption that all expected cell frequencies become large. However, this condition is not always satisfied and other asymptotic theories must be considered. For testing a specified simple hypothesis, Morris gave conditions for the asymptotic normality of the Pearson and likelihood ratio statistics when both the sample size and number of cells become large (even if the expected cell frequencies remain small). Monte Carlo techniques are used to examine the applicability of the normal approximations for moderate sample sizes with moderate numbers of cells.

KEY WORDS AND PERASES: Asymptotic approximations, Likelihood ratio statistic, Pearson statistics, Chi-squared. 


\section{Introduction}

The use of chi-squared tests for goodness of fit has become widespread since their Introduction by Karl Pearson in 1900. Trad1tional consideration of large sample properties has depended upon the assumption that all expected cell frequencies become large. It is our contention that in many applications cell selection is dependent upon the sample size in such a way as to violate these traditional asymptotic assumptions. In this paper we explore the practical importance of recent results of Morris as they relate to this statistical question.

Let $\left(N_{1}, N_{2}, \ldots, N_{k}\right)$ be a multinomial random vector with proba$\underset{k}{\text { bility parameter }} \underset{\sim}{p}=\left(p_{1}, p_{2}, \ldots, p_{k}\right)$ such that $n=\sum_{i=1}^{k} N_{1}$ and $1=\sum_{i=1} p_{i}$. Consider the problem of testing the null hypothesis $H_{0}: \underline{P}=q$, for some completely specifled probability vector $q$, against all possible alternatives. The test most frequently used is the one suggested by Karl Pearson (1900) which rejects $\mathrm{H}_{0}$ for sufficiently large values of

$$
x_{k}^{2}=\sum_{1=1}^{k}\left(N_{1}-n q_{1}\right)^{2} / n q_{1}
$$

Th1s stat1st1c will be referred to as the Pearson goodness-of-fit statistic.

The use of the likelthood ratio test statistic was proposed by J. Neyman and E. Pearson (1926). The 1ikelihood ratio statistic rejects $\mathrm{H}_{0}$ for large values of

$$
G_{k}^{2}-2 \sum_{i=1}^{k} N_{1} \log \left(N_{1} / n q_{1}\right)
$$


This statistic has become more popular as the avallability of high-speed computers has increased.

When $\mathrm{H}_{0}$ is true, both statistics are well known to have the same limiting central chi-squared distribution under the traditional limiting argument which requires that $\min _{1 \leq i \leq k} n p_{1} \rightarrow \infty$ as $n \rightarrow \infty$. Therefore, when all expected frequencies are large the chi-squared distribution can be used to establish approximate critical regions for each test statistic. However, it is not uncommon in practice to use the sample size to determine the number of cells. Then the number of cells is generally increased when the sample 18 increased. In that case, both $x_{k}^{2}$ and $G_{k}^{2}$ can be shown to have asymptotic normal distributions under conditions which allow both $n$ and $k$ to become large without necessarily requiring that $\min _{1<i<k} \mathrm{np}_{i} \rightarrow \infty$. These conditions are reviewed in Section 2. Monte Carlo methods are used in Sections 4 and 5 to assess accuracy of the asymptotic normal and chi-squared approximations to the distributions of the Pearson and likelthood ratio test statistics for moderate numbers of cells and moderate sample sizes.

\section{2: Asymptotic Normality}

Several authors have demonstrated the asymptotic normality of certain goodness-of-fit statistics under conditions which do not require that all expected frequencles become large as the sample size increases.

The number of cells must increase with the sample size. A simple example is the test for a uniform distribution on a fixed interval where the interval is partitioned into a number of subintervals of equal length. If it is desired to achleve a specified expected frequency 
$\lambda$ for each subinterval, then $k$ subintervals are used for a sample size of $n$, where $k$ is selected to make $n / k$ close to $\lambda$. If $n$ is Increased $k$ would also be increased.

This leads to the consideration of the limiting distributions of goodness-of-fit statistics for sequences of multinomials of increasing dimension. Consider the sequence of multinomial random vectors

$$
\left\{\left(N_{1, k(1)}, N_{2, k(1)}, \ldots, N_{k(1), k(1)}\right)\right\}_{1=1}^{\infty}
$$

where the 1 -th vector in the sequence has $k(1)$ cells, with sample size $n_{k}=\sum_{j=1}^{k} N_{j, k}$ and probability vector $\left(p_{1 k}, p_{2 k}, \ldots, p_{k k}\right)$ with $1=\sum_{j=1}^{k} p_{j k} \cdot$ (The underlying subscript 1 is hereafter suppressed to simplify notation.) We will require the sample size $n_{k}$ to increase as $k$ increases. Since the asymptotic moments for the statistics are derived from independent Poisson frequencies we need to define a corresponding sequence of Poisson random vectors. For each multinomial vector $\left(N_{1 k}, N_{2 k}, \ldots, N_{k k}\right)$, let $\left(Y_{1 k}, Y_{2 k}, \ldots, Y_{k k}\right)$ be a vector of Independent Polsson random varlables such that $E\left(Y_{1 k}\right)=E\left(N_{1 k}\right)$.

Morris (1966, 1975) generalized a conditioning argument given by Steck (1957) to obtain a central limit theorem for sums of functions of multinomial frequencies. The method requires that the sum of functions of independent Poisson frequencies has a limiting normal distribution. Then under mild conditions the asymptotic normality of the sum under the multinomial distribution can be obtained by conditioning on the sum of the independent Poisson frequencies.

As special cases, Morris (1975) derived central limit theorems for the Pearson and likelihood ratio statistics. Although asymptotic normality is valid for certain classes of alternatives we only consider the case 
where the null hypothesis is true in this paper. In that case sufficlent conditions for asymptotic normality as $k+\infty$ are

$$
\text { (1) } \max _{1 \leq 1 \leq k} P_{1 k}=O(1) \text { as } k+\infty \quad \text { and }
$$

(ii) $n_{k} p_{i k}$ is uniformly bounded below by some constant.

These conditions are not necessary and other sets of sufficient conditions have been given by Steck (1957) and Holst (1972, 1976).

When the null hypothesis is true the asymptotic mean and varlance for the Pearson statistic are given by

$$
\mu_{p, k}=k
$$

and

$$
\sigma_{P, k}^{2}=2 k+\sum_{j=1}^{k}\left(1-k^{-1} p_{j k}\right) / n_{k} p_{j k}
$$

However, it can be shown that Morris's central limit theorem for the Pearson statistic 18 valid when $\mu_{p, k}$ and $\sigma_{P, k}^{2}$ are replaced by the corresponding exact moments. Exact moments for the Pearson statistic were derived by Haldane (1937) and it is easily seen that

$$
E\left(x_{k}^{2}\right)=\mu_{P, k}-1
$$

and

$$
\operatorname{Var}\left(x_{k}^{2}\right)=\sigma_{p, k}^{2}-2\left[1+\frac{k-1}{n_{k}}\right] \text {. }
$$

The effect on the accuracy of the normal approximation from replacing $\mu_{\mathrm{P}, \mathrm{k}}$ and $\sigma_{\mathrm{P}, \mathrm{k}}^{2}$ by the exact values is examined in Sections 4 and 5 . Note that $\sigma_{\mathrm{p}, \mathrm{k}}^{2}$, and consequently $\operatorname{Var}\left(\mathrm{x}_{\mathrm{k}}^{2}\right)$, can be much larger than the chi-squared variance on $k .-1$ degrees of freedom when the expected frequencles are not all equal. 
The asymptotic moments for the likellhood ratio statistic are also derived from independent Poisson random variables. Define the Poisson Information kernel by

$$
I(y, m)=\left\{\begin{array}{ll}
y \log (y / m)-y+m, & \text { if } y>0 \\
m, & \text { if } y=0
\end{array} .\right.
$$

Then the first two asymptotic moments are given by

$$
\mu_{L R, k}=\sum_{j=1}^{k} E\left[I\left(Y_{j k}, n_{k} p_{j k}\right)\right]
$$

and

$$
\sigma_{L R, k}^{2}=\underbrace{k}_{j=1} \operatorname{Var}\left[I\left(Y_{j k}, n_{k} p_{j k}\right)\right]-n_{k} \gamma_{k}^{2}
$$

where

$$
Y_{k}=\frac{2}{n_{k}} \sum_{j=1}^{k} \operatorname{Cov}\left[I\left(Y_{j k}, n_{k} P_{j k}\right), Y_{j k}\right]
$$

An examination of these asymptotic moments is useful in determining when the asymptotic chi-squared approximation is appropriate. A graph of $E[I(Y, m)]$ is presented in Figure $A$ for a Poisson random variable $Y$ with mean $m$. The rapid decline of $E[I(Y, m)]$ as $m \rightarrow 0$ indicates that $\mu_{L R, k}$ can be much smaller than the chi-squared mean when many expected frequencles are smaller than one-half. However, the graph also shows that $\mu_{L R, k}$ is substantially larger than $k-1$ when most expected frequencies are between one and five. The mean of the likelihood ratio statistic is close to $k-1$ when almost all expected frequencles are large. 
The graphs of $[\operatorname{Var} I(Y, m)]$ and $\operatorname{Cov}[I(Y, m), Y]$ presented in Figures $B$ and $C$ give a good Indication of the behavior of $\sigma_{L R, k}^{2}$. The asymptotic variance can be much smaller than $2(k-1)$ when most expected frequencles are smaller than one, but it is larger than $2(k-1)$ when most expected frequencles are moderate. These figures Indicate that the chi-squared approximation for the likelihood ratio statistic may give inflated critical levels when most expected frequencies are moderate and extremely conservative critical levels when most expected frequencies are smaller than one-half.

- Insert Figures $B$ and $C$ about here --

It is interesting to note that Pearson and 11kelihood ratio statistics have different Iimiting normal distributions as $k+\infty$. The difference in behavior is largely due to the differing influence given to very small observed counts by the statistics. This effect was described by Larntz (1978) for expected frequencles in the range of 2.0-5.0. Here we examine the effect for smaller expected frequencies. Table 1 illustrates the general pattern. For a cell with an expected frequency larger than one an observed count of zero or one makes a larger minimum contribution to $G_{k}^{2}$ than $x_{k}^{2}$. Consequently, when most expected cell frequencies are in the range of 1.0-5.0 the first two moments for $G_{k}^{2}$ are larger than those for $x_{k}^{2}$. However, the contribution to $x_{k}^{2}$ for a nonzero count can be quite large when the expected frequency 1s. less than one, and the first two moments for $x_{k}^{2}$ are larger than the corresponding moments for $G_{k}^{2}$ when a sufficient number of expected frequencies are less than one. 


\section{Monte Carlo Procedures}

A Monte Carlo study was performed to assess the accuracy of the asymptotic chi-squared and normal approximations for moderate cell sizes when most expected frequencies do not exceed five. The objectives were (a) to determine when the normal approximation is sufficiently more accurate to justify the additional computation, (b) to examine how the accuracy of the asymptotic approximations is affected by departures from the conditions Imposed by the central limit theorems, and (c) to determine when the use of exact means and varlances provides better normal approximations.

In this study values of $x_{k}^{2}$ and $G_{k}^{2}$ were simulated for multinomials with $3,4,10,40,100,400$, and 1000 cells. For each cell size, sample sizes were selected such that $\lambda=\mathfrak{n}_{k} / k$ achieved the values $1 / 4,1 / 2$, $1,2,3$, and 5 as closely as possible. Some cases with 400 and 1000 cells were omitted because of the extreme computational cost. For each of the nine null hypotheses selected and each combination of $\lambda$ and $k$, 2500 multinomial random vectors were simulated. Each multinomial vector was used to produce a value for $x_{k}^{2}$ and $G_{k}^{2}$. Therefore, the $x_{k}^{2}$ and $G_{k}^{2}$ values are correlated. ${ }^{l}$

${ }^{1}$ Computations were performed using FORTRAN programs on a CDC 6600 computer. "Multinomials were generated from uniform random numbers by classifying the uniforms into $k$ categories [see Koehler (1977)]. The uniform random numbers were produced by a multiplicative congruential generator using modulus $2^{47}$ and multiplier $5^{17}$. 
Denote the probability simplex by

$$
\begin{array}{r}
T_{k}={\underset{\sim q}{p_{k}} \in R^{k}:}_{\sum_{1=1}^{k}}^{k} p_{1 k}=1 \text { and } p_{1 k} \geq 0 \\
\quad \text { for all } 1 \leq 1 \leq k\} .
\end{array}
$$

and let

$$
\left.T_{k}^{+}={\left\{p_{k}\right.}_{2} \in T_{k}: p_{1 k} \geq p_{2 k} \geq \cdots \geq p_{k k}\right\}
$$

Any point in $T_{k}$ can be obtained from a permutation of the coordinates of some point in $\mathrm{T}_{k}^{+}$; therefore only null hypotheses in $\mathrm{T}_{k}^{+}$need be considered. The nine null hypotheses examined in this study are labeled In Table 2. Null hypothesis 1 is the center of $T_{k}$ and will be referred to as the hypothesis of symmetry. The other points were selected to cover a wide range of $\mathrm{T}_{k}^{+}$. Hypothesis 5 is the center of gravity of $\mathrm{T}_{k}^{+}$ when mass is uniformly distributed over $\mathrm{T}_{k}^{+}$.

The accuracy of the asymptotic normal approximation was examined for three standardized versions of $x_{k}^{2}$ and $G_{k}^{2}$. The first two exact moments for $x_{k}^{2}$ were computed directly, but the first two exact moments for $G_{k}^{2}$ were estimated by a Monte Carlo procedure which uses $x_{k}^{2}$ as a control varlate. The standardized statistics are denoted by the following symbols. $P_{E}-x_{k}^{2}$ standardized with $E\left(x_{k}^{2}\right)$ and $\operatorname{Var}\left(x_{k}^{2}\right)$. $L R_{E}-G_{k}^{2}$ standardized with Monte Carlo estimates of the exact mean and standard deviation. $P_{A}--x_{k}^{2}$ standardized with $\mu_{P, k}$ and $\sigma_{P, k}^{2}$. $L R_{A}-G_{k}^{2}$ standardized with $\mu_{L R, k}$ and $\sigma_{L R, k}^{2}$. $P_{C}-x_{k}^{2}$ standardized with the mean and standard deviation of a chi-square random varlable with 


$$
\begin{gathered}
k-1 \text { degrees of freedom. } \\
L R_{C}--G_{k}^{2} \text { standardized in the same manner as } P_{C} \text {. }
\end{gathered}
$$

Since a standardized chi-squared random variable converges in distribution to a standard normal random variable as the degrees of freedom increase, $P_{C}$ and $L_{C}$ will be well approximated by the standard normal distribution for large $k$ when the chi-squared distribution provides an adequate approximation for the distribution of $x_{k}^{2}$ and $G_{k}^{2}$ respectively. Rejection levels and percentiles were simulated for all six of the standardized statistics for nominal levels, .001, .005, .01, .025, .05, $.1(.1) .9, .95, .975, .99, .995, .999$. Complete tables for the .01 and .05 levels are avaflable from the authors. Some special cases are presented in the next two sections to illustrate general trends.

\section{The Symmetrical Case}

Small sample properties of goodness-of-fit statistics have been most frequently studied for the null hypothesis of equal cell probabilities. One reason is that many goodness-of-fit problems can be transformed into the problem of assessing the goodness-of-fit of the uniform distribution on the unit interval and in that case it is reasonable to select cells of equal widths. Second, the computation, of exact probab1lity levels is relatively simple since $X_{k}^{2}$ and $G_{k}^{2}$ are invariant under permutations of the observed frequencies when all cell probabilities are equal. In this section we examine the distributions of $x_{k}^{2}$ and $G_{k}^{2}$ under the nul1 hypothesis of symmetry.

Exact probability levels for the Pearson statistic have been examined by Vessereau (1958), Nass (1958), Slakter (1966), Good, et. al (1970), 
Zahn and Roberts (1971), and Katti (1973) for small numbers of cells with equal expectations. Their consensus opinton is that the traditional ch1-squared approximation does not introduce serious absolute errors at nominal levels .05 and .01 in the upper tail when $n \geq 10$. Zahn and Roberts recommend that $n \geq 25$ when the chi-squared approximation is used at similar nominal levels in the lower tail.

Citing Verrereau's work some authors have suggested that an appropriate rule for deciding when the chi-squared approximation for $x_{k}^{2}$ is adequate Is to use the chi-squared approximation whenever $n \geq n_{0}$, where $n_{0}$ is a fixed positive integer. However, any fixed $n_{0}$ will be inadequate when $k$ is sufficiently large. A more appropriate criterion is to require $\mathrm{n}^{2} / \mathrm{k}>\mathrm{c}$ for some constant $c$. Unless $\mathrm{n}^{2} / \mathrm{k}$ is sufficiently large, the Pearson statistic will have a high probability of assuming its minimum value and w11l not allow for an adequate continuous approximation. Our Monte Carlo results indicate that the chi-squared approximation is reasonably adequate for the symmetrical case when $k \geq 3, n \geq 10$, and $n^{2} / k \geq 10$

It should be noted that $\mathrm{n}^{2} / \mathrm{k} \rightarrow \infty$ is a necessary condition for $x_{k}^{2}$ to have a limiting normal distribution as $k \rightarrow \infty$ under any null hypothesis. Therefore, the rule $n^{2} / k>c$ is also an appropriate guideline for the application of the normal approximation.

The distribution of the likelihood ratio statistic is generally not well approximated by the chi-squared distribution when $\lambda \leq 5$. Unlike the moments of $x_{k}^{2}$, the mean and variance of $G_{k}^{2}$ do not closely match the corresponding moments of the chi-squared distribution with $k-1$ degrees of freedom. As noted in Section 2, the mean and variance of $G_{k}^{2}$ 
are smaller than the chi-squared moments when $\lambda<0.5$ and larger when $\lambda>1$. Hence the chi-squared approximation produces conservative critical levels in the first case and liberal critical levels in the latter case. The simulated critical levels for ${ }^{2} R_{C}$ presented in Figures $D, E, F$, and $G$ Illustrate how extremely inaccurate the chi-squared approximation can be when the number of cells is moderately large. When $\lambda=0.5,-H_{L R, k}=(1.007) k$ and $\sigma_{L R, k}^{2}=(.56) k$ and the estimated critical level drops to 0.0024 at $k=100$ for the .05 nominal level. When $\lambda=1$, the estimated critical level is .9776 at $k=1000$ for the .05 nominal level. Critical levels are most liberal when $\lambda$ is close to 2, but even when $\lambda=5$ the estimated critical level is 0.126 at $k=100$ for the .05 nominal level.

The Inadequacy of the chl-squared approximation was previously noticed by Good, et. al. (1970) who stated that "The distribution of the likelihood ratio statistic is by no means as well approximated by the chi-squared distribution as that of $x^{2}$ when $n / k<1 . "$ Lamtz (1978) observed that the likelihood ratio statistic ylelds exact levels in excess of the nominal levels when the minimum expected frequencles are between 2 and 4. Fortunately the standard normal distribution provides a good approximation for the right tall of the $L_{A}$ distribution. The normal approximation for $L R_{A}$ is quite adequate at the .05 and .01 nominal levels when $k \geq 3$, $n \geq 15$, and $n^{2} / k>10^{\circ}$. Figures $D, E, F$, and $G$ show that the normal approximation is appreciably more accurate for $L R_{A}$ than $L R_{E}$ at the .01 nominal level. In addition, the normal approximation is generally more accurate for $L R_{A}$ than either $P_{A}$ or $P_{E}$ for nominal levels smaller than .05 and moderate values of $k$. The estimated rejection levels for 
$P_{A}$ and $P_{E}$ tend to be too large. The evaluation of the first four central moments indicates that the skewness converges to zero and the kurtosis converges to three faster for $L_{A}$ than for either $P_{A}$ or $P_{E}$ as $k \rightarrow \infty$.

- Insert Figures $D, E, F$, and $G$ about here --

Monte Carlo power comparisons showed that for the null hypothesis of symmetry, $x_{k}^{2}$ is slightly more powerful for near alternatives. The Pearson test is decidedly dominant as the alternative moves toward a boundry of $T_{k}$ which contains a high proportion of zeros and a few relatively large probabilities. The likelihood ratio test is dominant at alternatives which lie near boundrles of $T_{k}$ which contain a small proportion of near zero probabilities and have nearly equal probabilities in the remaining cells. This pattern agrees with observations made by West and Kempthorne (1971) from exact computations for 2, 3, and 4 cell examples. The boundries near which $G_{k}^{2}$ is dominant become close to the symmetrical null hypothesis in the Euclidean sense as $k$ becomes large, but the areas where $x_{k}^{2}$ is more powerful may not. This indicates that $x_{k}^{2}$ is more powerful than $G_{k}^{2}$ for a very large portion of the simplex when $k$ is moderately large.

\section{SOME UNSYMMETRICAL CASES}

General rules are more difficult to prescribe for unsymmetrical null hypotheses. In an extremely Influential paper, Cochran (1954) gave a set of recommendations for the use of the chi-squared approximation for the Pearson statistic which generally require most expected frequencies to be at least five but allow a few to be between one and five. Vessereau found Cochran's recommendations to be stringent for the cases he considered, but he noticed that the chi-squared approximation for the Pearson statistic tends 
to produce inflated critical regions when small unequal expected frequencles are present. This phenomenon was partially explained in Section 2 where it was noted that under a null hypothesis with many small, unequal frequencles the variance of $x_{k}^{2}$ can be much larger than $2(k-1)$ but the mean is $k-1$. Roscoe and Byars (1971) examined the chi-squared approximation for the Pearson statistic for the hypthesis of symmetry and two levels of skewness. Under their most extreme level of skewness they recommend that the chi-squared approximation be used at the .05 level only when $\lambda \geq 2$ and at the .01 level when $\lambda \geq 4$, where $\lambda=n / k$. The rule proposed by Roscoe and Byars works for their special cases, but in general no rule based solely on a minimum value of $\lambda$ can hold under all unsymmetrical null hypotheses.

- Insert Figures $B$ and $I$ about here ---

Monte Carlo rejection rates for all nine null hypothese are given in Figures $H$ and $I$. In this study null hypothese 2, 3, and 4 all have at least one cell probability which does not become small for large values of $k$, and at least k-2 cells with small, equal probabilities. The chi-squared approximation for $x_{k}^{2}$ also gives very liberal critical reglons under hypothesis 3 , but they are not quite as bad as those under hypothesis 2 . Hypothesis 4 is close enough to the center of the simplex so that the variance is not greatly Inflated and, therefore, the chi-squared approximation is reasonably accurate for $x_{k}^{2}$. These observations are supported by the summary of Monte Carlo results given in Figures $A$ and $B$ for the nominal .05 and .01 levels. Figures $J$ and $R$ show that the chi-squared approximation for $G_{k}^{2}$ gives very conservative critical levels under hypothesis 2 when $\lambda \leq 5$. For this case the varlance and mean of $G_{k}^{2}$ are smaller than the chi-squared moments. However, the general behavior of the ch1-squared approximation for $G_{k}^{2}$ is exhlbited under hypotheses 3 and 4 . For those hypotheses the $k-2$ smallest 
expected frequencies are 1dentical. The chi-squared approximation gives conservative critical levels when these expected frequencies do not exceed 0.5 and it gives quite liberal critical levels when these expected frequencies are between 1 and 5. The critical regions given by the chi-squared approximation are most liberal when these expected frequencies are near 2 and become Increasingly conservative when the expected frequencles are made smaller

\section{-- insert Figures $J$ and $K$ about here ---}

Figures $\mathrm{J}$ and $\mathrm{K}$ indicate that the normal approximation is much more accurate than the chi-squared approximation under null hypotheses 2 when $n^{2} / k$ is sufficiently large. Standardization by the asymptotic moments seems to be best. The normal approximations for $L R_{E}$ and $P_{E}$ tend to give critical levels which are too large at the .05 and .01 nominal levels. This result was also observed under hypotheses 3 and 4. It is interesting to note that the normal approximation is generally better for $L R_{A}$ than $P_{A}$. The $P_{A}$ critical levels tend to be too large, especially at the .01 nominal level. As in the symmetric case, the skewness and kurtosis tend to the normal values faster for ${ }^{L R} R_{A}$ than for $P_{A}$ as $k$ becomes large.

In general the normal approximation for $\mathrm{LR}_{\mathrm{A}}$ is less affected by the presence of one or two large cell probabilities than the normal approximation for $P_{A}$. The Monte Carlo results for null hypotheses 2, 3, 4 suggest that the normal approximation for $L_{A}$ are not seriously misleading if $k \geq 10$, $\mathrm{n} \geq 20$, and $\mathrm{n}^{2} / \mathrm{k} \geq 100$. These minimum values probably should be increased If a few cells contain more than ninety percent of the total probability. 
Hypotheses 5 through 9 share the common property that no two cell probabilities are equal. For hypothesis 6 the behavior of the likelihood ratio and Pearson statistics is simflar to their behavior under hypothesis 1 (symmetry) and hypothesis 4.

Null hypotheses 5, 7, 8, and 9 all have some very small expected frequencles. The presence of small expected frequencles has 1ittle effect on the normal approximation for $L R_{A}$, but the effect on the normal approximation for $P_{A}$ varies with the number of small expected frequencies. In general, the normal approximation for $L R_{A}$ is the most accurate at the .05 and .01 critical levels. As in previous cases, the chi-squared approximation: for $G_{k}^{2}$ 8lves conservative critical levels when most expected frequencies are smaller than 0.5 and liberal levels when most expected frequencies are between 1 and 5 . The ch1-squared approximation for $x_{k}^{2}$ yields liberal critical levels when most expected frequencies are less than one.

Unlike the other cases, the presence of an extremely small expected frequency can cause the normal approximation for $P_{A}$ and $P_{E}$ to give very conservative critical reglons. This is most dramatically 1llustrated by the estimated critical levels for hypothesis 9 presented in Figures L and and $M$. This hypothesis is close to hypothesis 1 in the sense that every cell but one has an expected frequency larger than $(0.9) \lambda$. The $k$-th cell has.expected freqeuncy $(.1 \lambda) / k$. Hence the conditional distribution of $x_{k}^{2}$ given that $\mathrm{N}_{\mathrm{kk}}=0$ is well approximated by a chi-squared distribution with k-2 degrees of freedom. Furthermore, the probab1lity that $\mathbb{N}_{k k}=0$ is $\left(1-.1 k^{-2}\right)^{k}$, which converges to $I$ as $k \rightarrow \infty$. Hence the distribution of $\mathrm{x}_{k}^{2}$ severely deviates from the chi-squared distribution only in very extreme regions of the upper tail. However, the infrequent non-zero values of $\mathrm{N}_{\mathrm{kk}}$ 
$\sigma_{P, k}^{2}$ and $\operatorname{Var}\left(x_{k}^{2}\right)$ to be much larger than $2(k-2)$. Therefore, the normal approximate for $P_{A}$ and $P_{E}$ is conservative at commonly used critical levels, but the chi-squared approximations with k-2 degrees of freedom is quite adequate.

- Insert Figures $L$ and $M$ about here -

In general it was found that the normal approximation was more accurate for $L R_{A}$ than for $P_{A}$. This is 1llustrated by the results in Figures $H$ and $I$. In fact, the normal approximation for $L R_{A}$ is even accurate when several very small expected frequencles are present.

Monte Carlo power computations show that for ansymmetrical null hypotheses, either test may be dominant. The area of dominance for the Pearson statistic is generally not nearly as broad as it is for the symetrical null hypothesis case. In fact for some null hypotheses the likelihood ratio test completely dominates the Pearson test along specific directions.

As previously noted, Morris's central limit theorems are valid for a certain class of alternatives. Therefore, the normal approximations for $\mathrm{X}_{k}^{2}$ and $G_{k}^{2}$ provide computationally inexpensive power approximations. However, Monte Carlo results Indicate that it is not uncommon for these power approximations to be too large by as much as $20 \%$ for moderate power and moderate cell sizes. The discrepancy is generally smaller for $G_{k}^{2}$ than for $x_{k}^{2}$.

6. SUMMARY AND RECOMMENDATIONS

Clearly for the null hypothesis of symmetry, the chi-squared approximation for the Pearson statistic is quite adequate at the .05 and .01 nominal levels for expected frequencies as low as .25 when $k \geq 3$, 
$n \geq 10, n^{2} / k \geq 10$. The ch1-squared approximation 18 generally easier to apply than the normal approximation since the former procedure does not require the calculation of a mean and standard deviation. Furthermore, the theoretical results of Holst, Morris and Stein and the numerical results summarized in Section 6 Indicate that the Pearson test has some optimal local power properties in the symmetrical case when the number of cells is moderately large. Hence the Pearson goodness-of-fit test based on the traditional chi-squared approximation is preferred for the test of symetry.

In general, the normal approximation for $L R_{A}$ produces the most accurate critical reglons for unsymmetrical hypotheses. The Monte Carlo results for null hypotheses $5,6,7,8$, and 9 suggest that the use of this approximation will not be serlously misleading for a wide range of null hypotheses in the interfor of the simplex when $n \geq 15, n^{2} / k \geq 10$ and $k$ is selected so that most expected frequencles are less than 5 . Unlike the normal approximations for $P_{A}$ and $P_{E}$, the accuracy of the normal approximations for $L R_{A}$ is not seriously affected by the presence of a few extremely small expected frequencies. The chi-squared approximation for the Pearson statistic produces inflated rejection levels for unsymmetrical null hypotheses which contain many expected frequencles smaller than one.

The $C_{(m)}$ approximation for the Pearson statistic for the case of just a few small expected frequencies was proposed by Cochran (1946) and further studied by Yarnold (1970). The application of this approximation is limited to the cases for one and two small expected frequencies covered by the tables of percentage points given in Cochran's paper. Use of the normal approximation for $L R_{A}$ eliminates the need for extensive tables of the $C_{(m)}$ approximation. 
Modern computer programs which provide values of $G_{k}^{2}$ would have 11ttle trouble in providing values of $\operatorname{LR}_{A}$. These values can be compared to readily available tables of the percentlles of the standard normal distribution. 
$-20-$

?

FIGURE A

EXPECTED VALUE OF THE

POISSON INFORMATION KERNEL

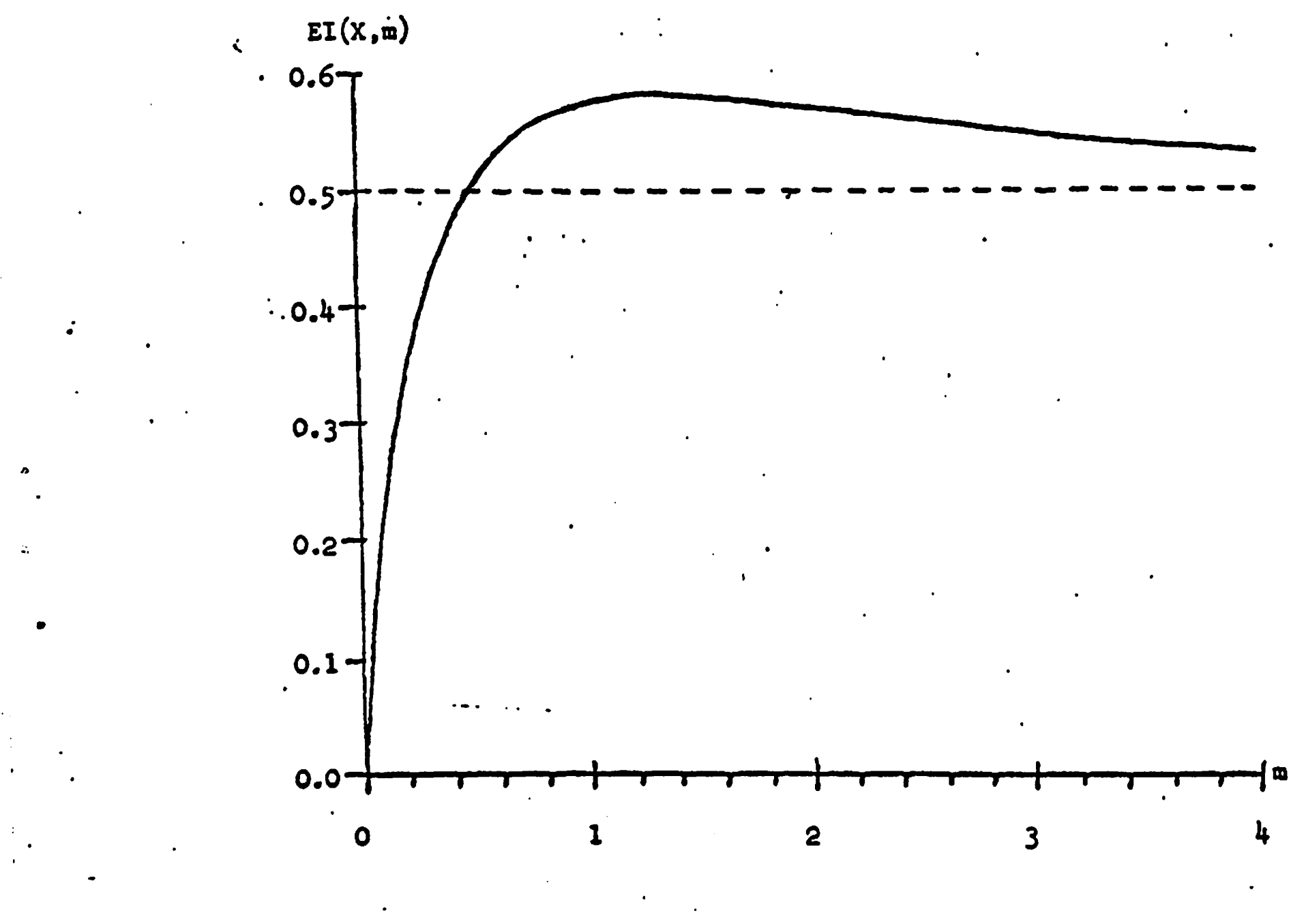


$-21-$

FIGURE B

VARIANCE OF THE

POISSON INFORMATION MODEL

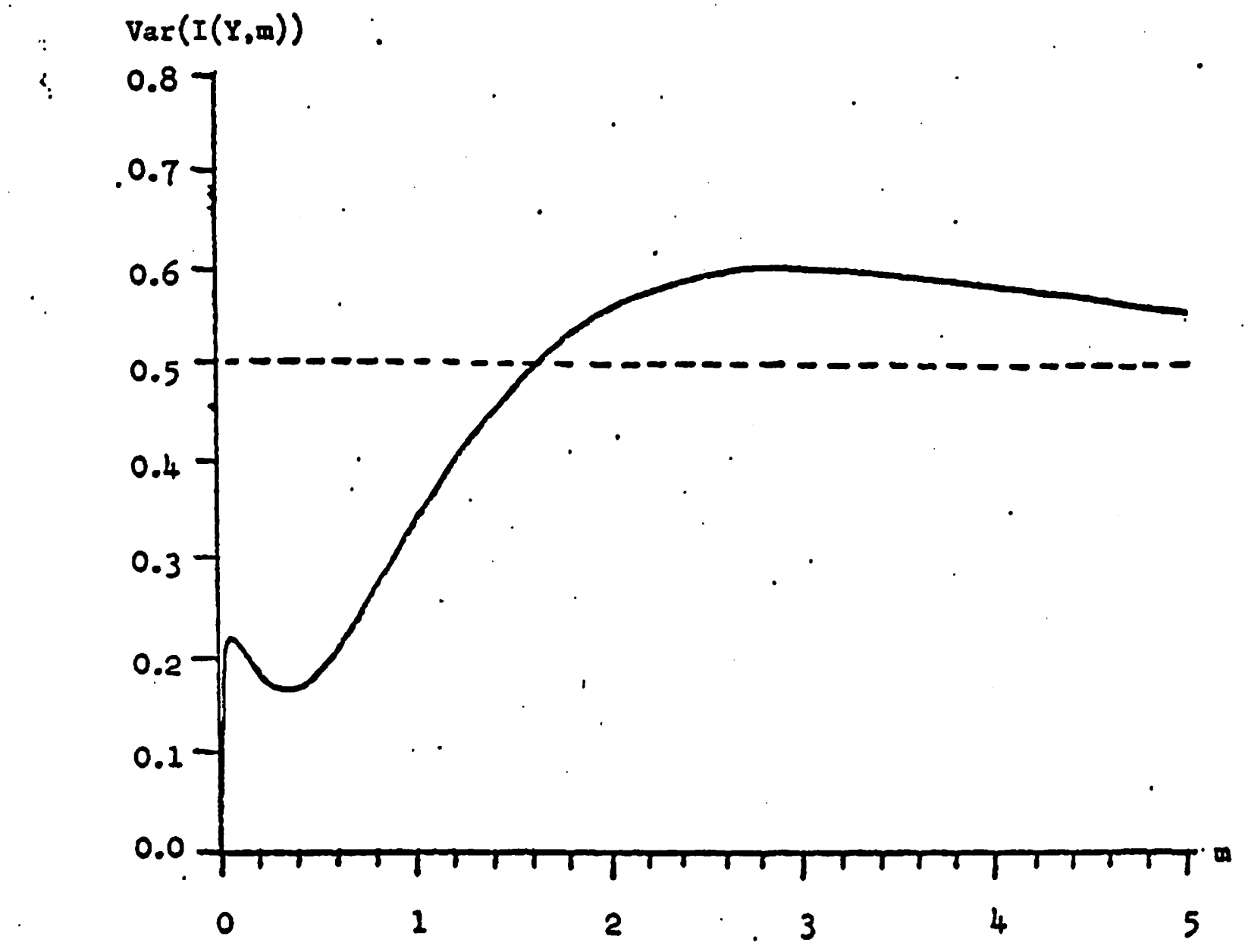


$-22-$

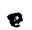

FIGURE C.

$2 \quad \cdot$
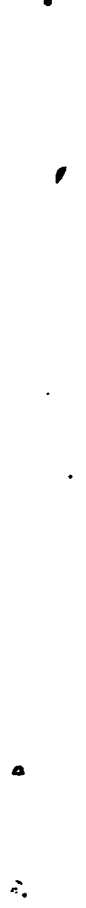

a
COVARIANCE BETWEEN Y AND THE

POISSON INFORMATION KERNEL

$\operatorname{Cov}[I(Y, m) Y]$

$\therefore \quad 0.207$

.

0.20
0.15

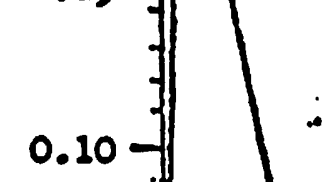

.

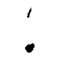

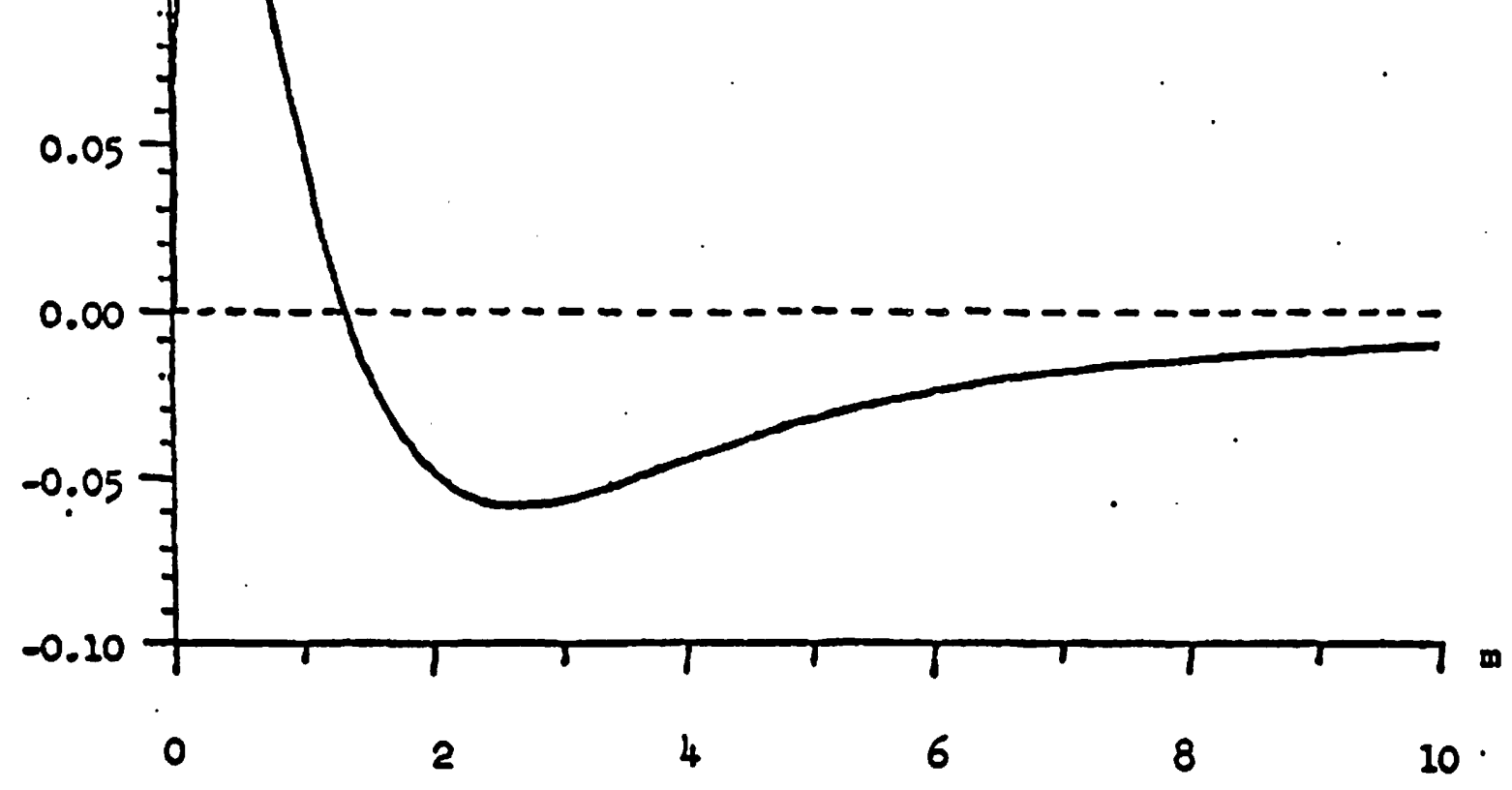

w

2

8

10 
FIGURE D

Estimated Probability of Excecdlng $2.95=1.645$

Under Hypothesis 1 when $n=.5 k$

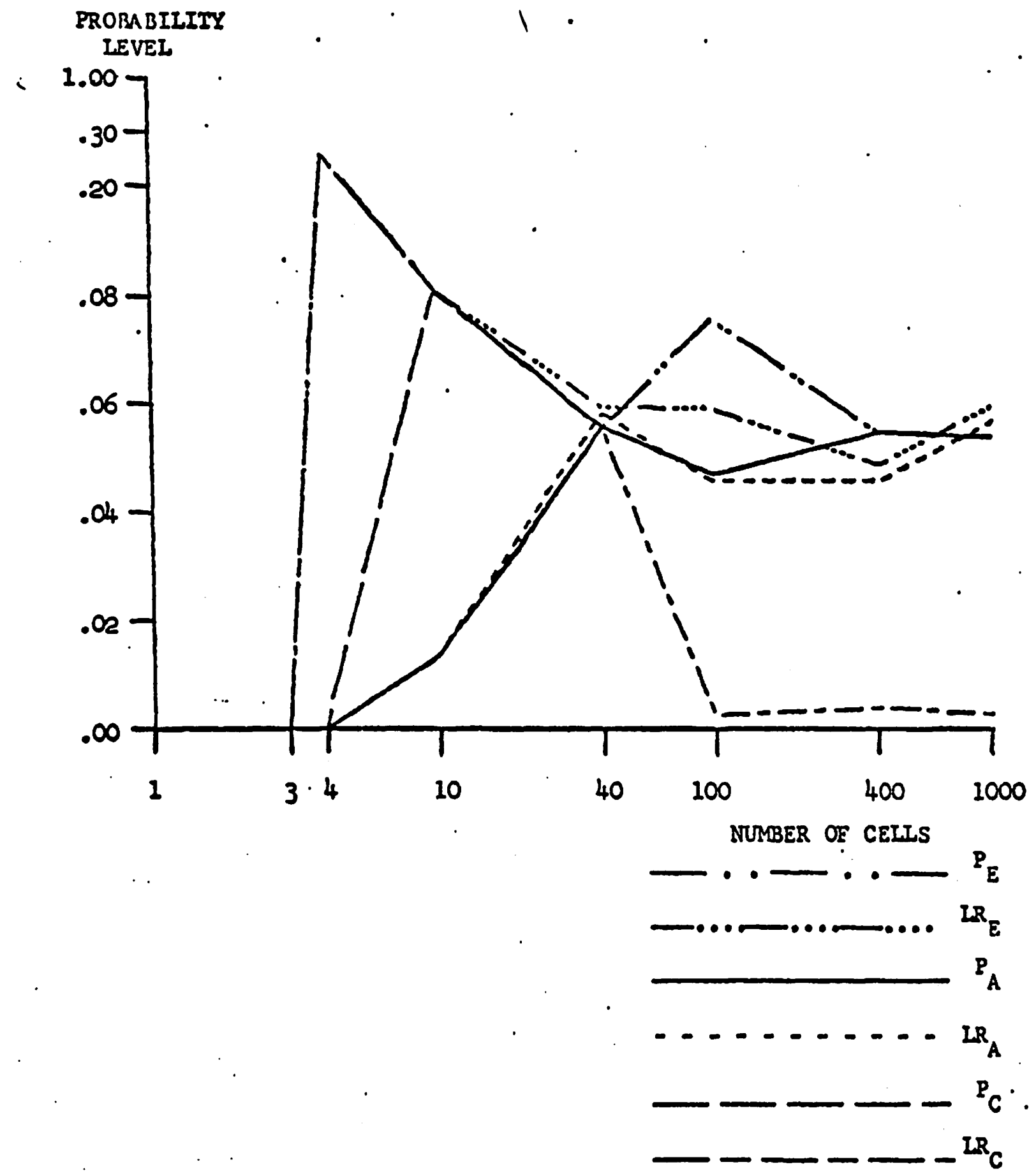


FIGURE E

Estimated Probability of Exceeding $Z_{.99}=2.326$

PROBAEILITY

Under Hypothest?s 1 when $n=.5 k$

LEVEI

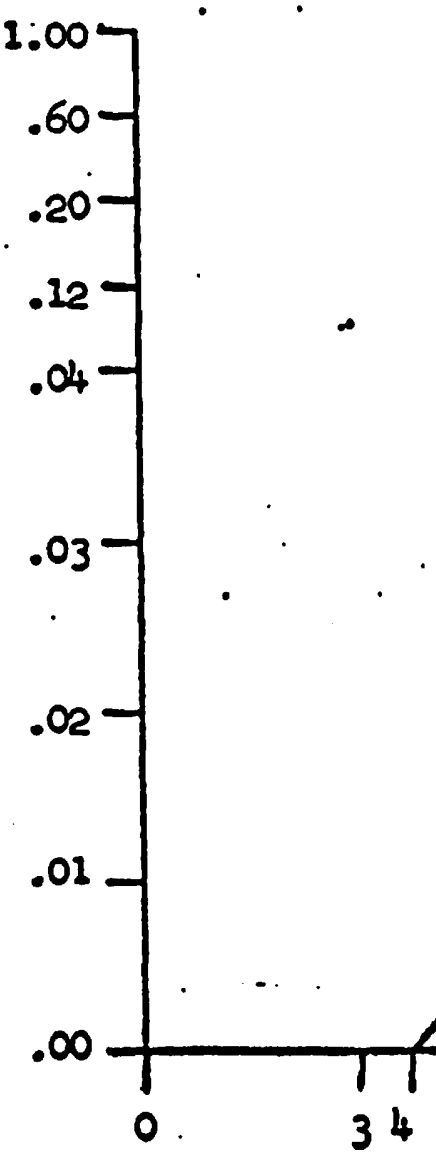

NURBER OF CELIS

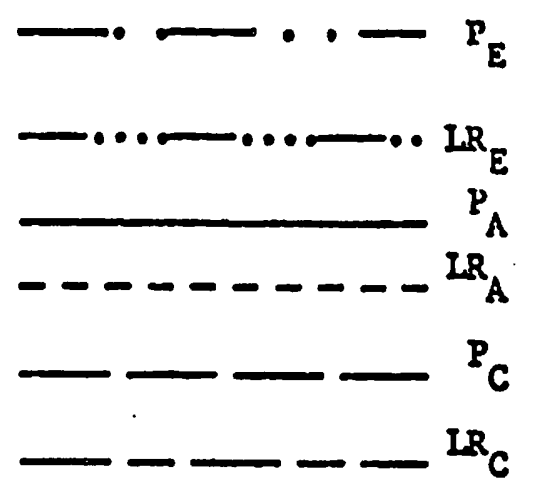




\section{FIGURE F}

Estimated Probability of Exceeding $2.95=1.645$

: PROBABILIIY Under Hypothes 1s 1 when $n$ a $k$

LEVEL
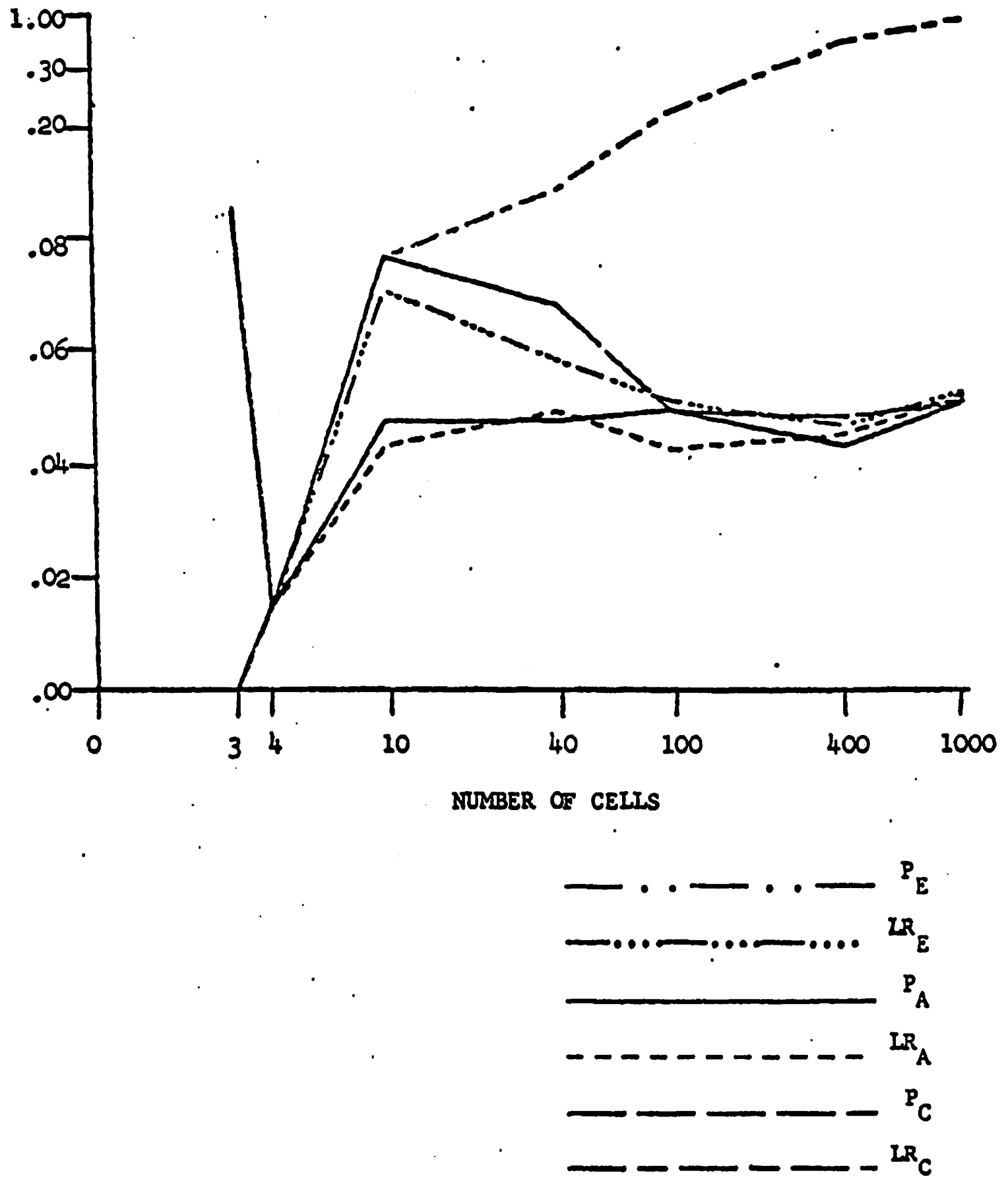
FIGURE G

Estimated Probability of Exceeding Z.99=2.326

Under Hypothesis 1 , when $n=k$

PROBABILITY

IEVEL
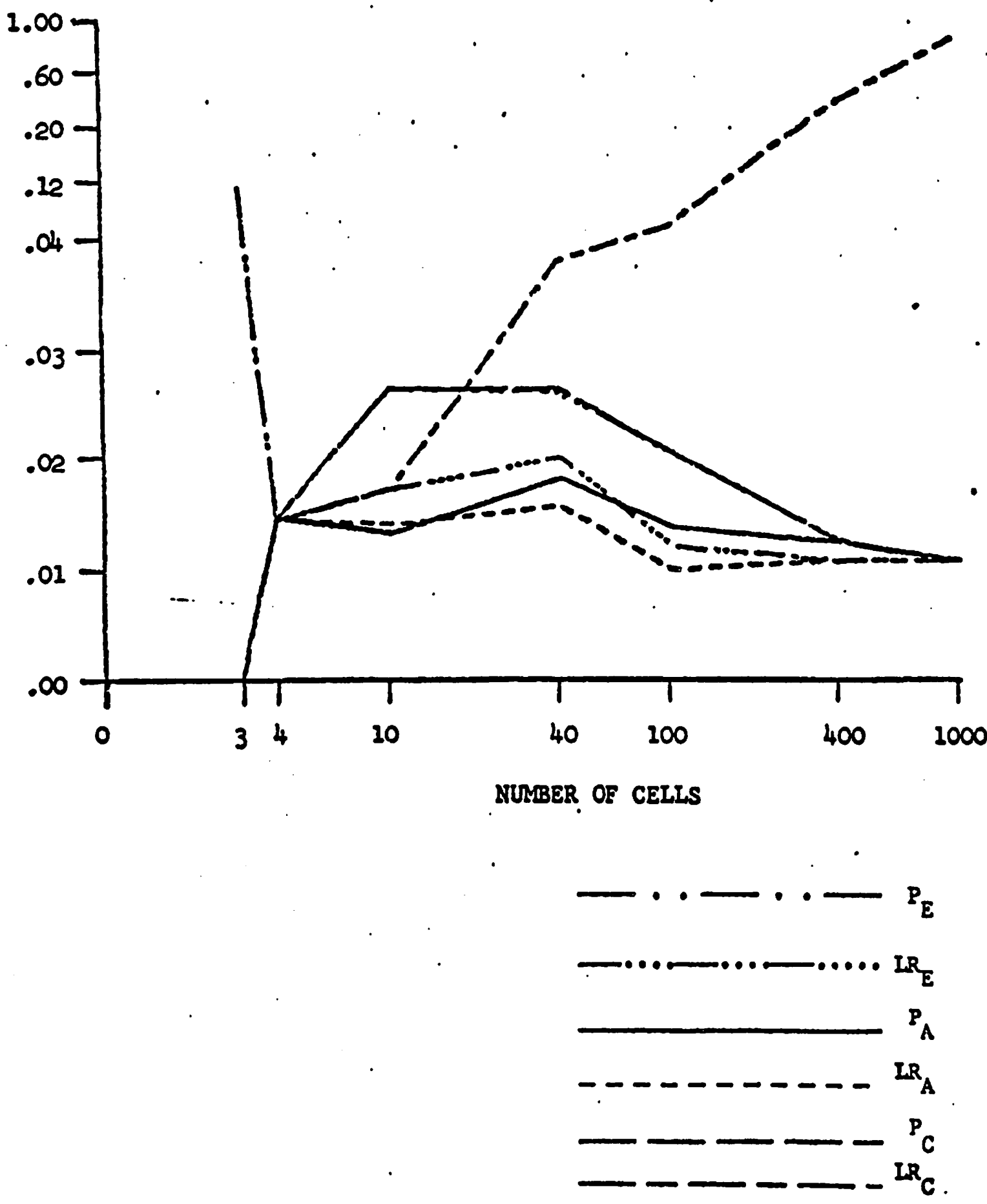
H. Monte Carlo Rejection Levels for the Nominal .05 Level

$\begin{array}{cccccc}P_{E} & I R_{E} & P_{A} & I R_{A} & P_{C} & L R_{C}\end{array}$ $\begin{array}{lllllll}\text { Hypothesis } & 123456789 & 123456789 & 123456789 & 123456789 & 123456789 & 123456789\end{array}$

\begin{tabular}{|c|c|c|c|c|c|c|c|}
\hline$\lambda=.25$ & $\begin{array}{r}3 \\
4 \\
10 \\
40 \\
100 \\
400\end{array}$ & $\begin{array}{lr}x 60000 & 1 \\
x 700 & 0310 \\
0260 & 10 \\
8776 & 6330 \\
866 & 330 \\
6 & 36330\end{array}$ & $\begin{array}{cc}x 60000 & 1 \\
x 700 & 0310 \\
02806971 & 6 \\
7786 & 6 \\
8866 & 3 \\
3 & 3\end{array}$ & $\begin{array}{lrl}060000 & 1 \\
0700 & 0310 \\
0261 & 0 & 20 \\
27 & & 330 \\
& & 330 \\
6 & 3 & 330\end{array}$ & $\begin{array}{lll}060000 & 1 \\
0700 & 0310 \\
0371 & 0371 \\
7 & \end{array}$ & $\begin{array}{ll}060000 & 1 \\
0700 & 0310 \\
0001000 & 1 \\
80006006 & 0 \\
80070 \quad 066 \\
087000\end{array}$ & $\begin{array}{l}060000 \quad 1 \\
070000310 \\
000000000 \\
000000000 \\
000000000 \\
000000000\end{array}$ \\
\hline$\lambda=.50$ & $\begin{array}{r}3 \\
4 \\
10 \\
40 \\
100 \\
400\end{array}$ & $\begin{array}{rrr}002819782 \\
0039 & 1 \\
86 & 77 & 30 \\
766 & 7 & 0 \\
76 & 6 & 30 \\
7 & 6 & 330 \\
6 & 6 & 330\end{array}$ & $\begin{array}{rc}000880782 \\
0009 & 081 \\
8677 & 6762 \\
66 & 6893 \\
777 & 9\end{array}$ & 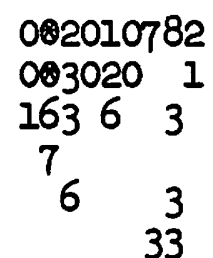 & 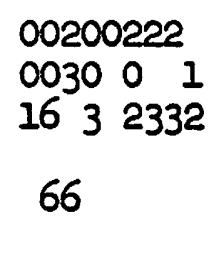 & $\begin{array}{c}082010782 \\
00308081 \\
80806880 \\
0060600 \\
078600 \\
070600\end{array}$ & $\begin{array}{c}002010226 \\
0030201 \\
10100000 \\
0000000 \\
000000006 \\
000000006\end{array}$ \\
\hline
\end{tabular}

$\lambda=1$

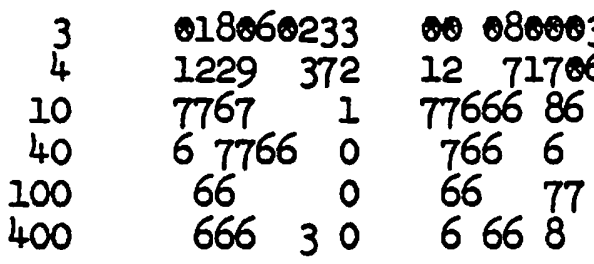
012060233 12111332
$32 \quad 31$ $\begin{array}{ll}6 & 0 \\ 6 & 0\end{array}$ 630
012330231 121131 1333
7 $\cdot 6$

00806003 01 80231 10261076 7096900 60970600 0960 0970

6779 366908 7877806 $\rightarrow 8 \div$ -7 10 7667006 69676099

70767 0768600 9769
109601

70273701

001010008 10000000 10000000

226000116 918060117 00706.027 2000000 10001000

82909923

०2087022 1907-027 00878000 - 00060000

$\lambda=5$

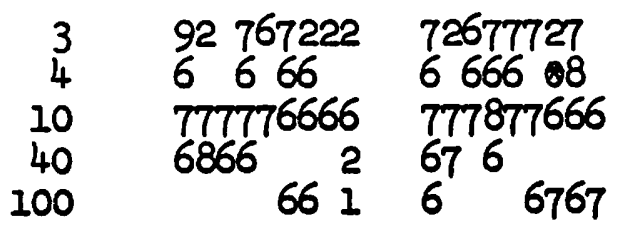

222232221 2 3222222

$$
\begin{aligned}
& 122333123 \\
& 233333133 \\
& 3 \quad 3
\end{aligned}
$$

จ2 766776 666800 707786099 68767 $6 \times 68680$
72987822

98787136 92099912 918079007 จ1 090009 
Code for Figure H.

Symbol

0
1
2
3
blank
6
7
8
9
0
0
$x$

Range for

Rejection Level

$$
\begin{aligned}
& 0-0.01 \\
& 0.01-0.02 \\
& 0.02-0.03 \\
& 0.03=0.04 \\
& 0.04=0.06 \\
& 0.06=0.07 \\
& 0.07=0.08 \\
& 0.08=0.09 \\
& 0.09=0.10 \\
& 0.10-0.20 \\
& 0.20-1.00 \\
& \text { No result - } \\
& \text { statistic assumes } \\
& \text { only one value }
\end{aligned}
$$


I. Monte Carlo Rejection Levels for the Nominal .01 Level

$\begin{array}{lllllll}\text { Nul1 } & P_{E} & L R_{E} & P_{A} & { }^{L R} & P_{C} & L R_{C}\end{array}$ $\begin{array}{lllllll}\text { Hypothesis } & 123456789 & 123456789 & 123456789 & 123456789 & 123456789 & 123456789\end{array}$

\begin{tabular}{|c|c|c|c|c|c|c|c|}
\hline$\lambda=.25$ & $\begin{array}{r}3 \\
4 \\
10 \\
40 \\
100 \\
400\end{array}$ & $\begin{array}{c}x+000008 \\
x 000006 \\
709861 \\
877988661 \\
66877670 \\
676677 \quad 0\end{array}$ & $\begin{array}{c}x 1000000 \\
x 0000086 \\
76696 \\
868667776 \\
9878666 \\
66662\end{array}$ & $\begin{array}{c}0800000 \\
080006 \\
799861 \\
877687661 \\
66777670 \\
676676 \quad 0\end{array}$ & $\begin{array}{l}01000000 \\
00000086 \\
76666 \\
666666 \\
66 \\
6 \quad 6\end{array}$ & $\begin{array}{c}00000080 \\
00000006 \\
00 \bullet 001 \\
800908007 \\
600907007 \\
60070700\end{array}$ & $\begin{array}{l}00000000 \\
000000 \\
010000100 \\
000000000 \\
000000000 \\
000000000\end{array}$ \\
\hline$\lambda=.50$ & $\begin{array}{r}3 \\
4 \\
10 \\
40 \\
100 \\
400\end{array}$ & $\begin{array}{c}0700777 \\
0007000 \\
6009792 \\
897788881 \\
697887770 \\
667660\end{array}$ & $\begin{array}{c}0700007 \\
007009 \\
6866 \\
6776699 \\
677669 \\
6\end{array}$ & $\begin{array}{rrr}0 & 70 & 0777 \\
0 & 02000 \\
72792 \\
696676881 \\
696786770 \\
667660\end{array}$ & $\begin{array}{ccc}0 & 70 & 0777 \\
0 & 007066 \\
6 & 66 \\
766 & 76 \\
666 & 66\end{array}$ & $\begin{array}{c}00700007 \\
00000000 \\
008000 \\
800708046 \\
600807006 \\
60060006\end{array}$ & $\begin{array}{l}0700117 \\
0002016 \\
002110010 \\
000000000 \\
000100000 \\
000000000\end{array}$ \\
\hline$\lambda=1$ & $\begin{array}{r}3 \\
4 \\
10 \\
40 \\
100 \\
400\end{array}$ & $\begin{array}{c}67900890 \\
76600987 \\
80878809 \\
808897880 \\
777676770 \\
66666660\end{array}$ & $\begin{array}{c}067909896 \\
7060696 \\
668 \quad 77076 \\
6 \otimes 866686 \\
7 \quad 88 \\
6 \quad 6 \quad 8\end{array}$ & $\begin{array}{c}062000890 \\
6 \quad 06887 \\
666699 \\
607686880 \\
7766770 \\
6666660\end{array}$ & $\begin{array}{cc}062020226 \\
6 & 6 \\
6 & 7 \\
666 & 66 \\
6 & 6 \\
6 & \end{array}$ & $\begin{array}{r}087900000 \\
76606000 \\
809608008 \\
800801006 \\
70060600 \\
0060600\end{array}$ & $\begin{array}{r}067900226 \\
667028 \\
667026 \\
001819007 \\
000010000 \\
000000000\end{array}$ \\
\hline$\lambda=2$ & $\begin{array}{r}3 \\
4 \\
10 \\
40 \\
100\end{array}$ & $\begin{array}{l}87009000 \\
809907010 \\
788908987 \\
78868697 \\
666686771\end{array}$ & 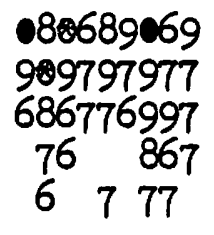 & $\begin{array}{ccc}28 & 201668 \\
607 & 6 & 6 \\
66676887 \\
687 & 7 & 97 \\
6 & 8 & 771\end{array}$ & $\begin{array}{cc}2 & 6^{221} \\
6 & 6 \\
6 & 6 \\
& 66\end{array}$ & $\begin{array}{l}007609000 \\
809706000 \\
700808000 \\
700606007 \\
60860600\end{array}$ & $\begin{array}{ll}0080 & 9 \\
110998 & 8 \\
006070129 \\
090 \\
0000 \\
000000\end{array}$ \\
\hline$\lambda=3$ & $\begin{array}{r}3 \\
4 \\
10 \\
40 \\
100\end{array}$ & $\begin{array}{l}70080088 \\
009099089 \\
709787008 \\
68777789 \\
686676891\end{array}$ & $\begin{array}{c}008080009 \\
807009080 \\
806797808 \\
68767977 \\
86668\end{array}$ & $\begin{array}{ccc}27 & 6 & \\
76 & 6 & 876 \\
606 & 7 & 808 \\
8767689 \\
76 & 7 & 881\end{array}$ & $\begin{array}{rrr}6 & 66 & 6 \\
& 6 & 66 \\
6 & 676 \\
6 & & 6\end{array}$ & $\begin{array}{l}709809009 \\
609808000 \\
700706000 \\
609708007 \\
608606006\end{array}$ & 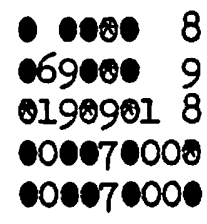 \\
\hline$\lambda=5$ & $\begin{array}{r}3 \\
4 \\
10 \\
40 \\
100\end{array}$ & $\begin{array}{l}879088787 \\
809789676 \\
989908980 \\
60677687 \\
676698\end{array}$ & $\begin{array}{l}870980780 \\
809888700 \\
897888979 \\
6866666 \\
66666767\end{array}$ & $\begin{array}{cc}6 & 676 \\
0 & 66 \\
687676869 \\
7666 & 87 \\
7 \quad 6688\end{array}$ & 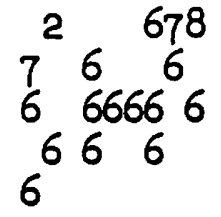 & $\begin{array}{l}879088780 \\
809788000 \\
800808080 \\
607796008 \\
6069606\end{array}$ & $\begin{array}{l}600067 \% \\
000068 \\
910089018 \\
010080009\end{array}$ \\
\hline
\end{tabular}


Code for Figure I.

Symbol

0
1
2
blank
6
7
8
9
0
0
$x$

Range for Rejection Level
$0-0.0005$
$0.0005-0.0025$
$0.0025-0.0050$
$0.0050-0.0150$
$0.0150-0.0200$
$0.0200-0.0250$
$0.0250-0.0300$
$0.0300-0.0350$
$0.0350-0.0500$
$0.0500-1.0000$
lio result
statistic assumes
only one velue 
FIGURE J

Estimated Probablitity of Exceeding $2.95=1.045$

Under Hypothes is $2, n=k$

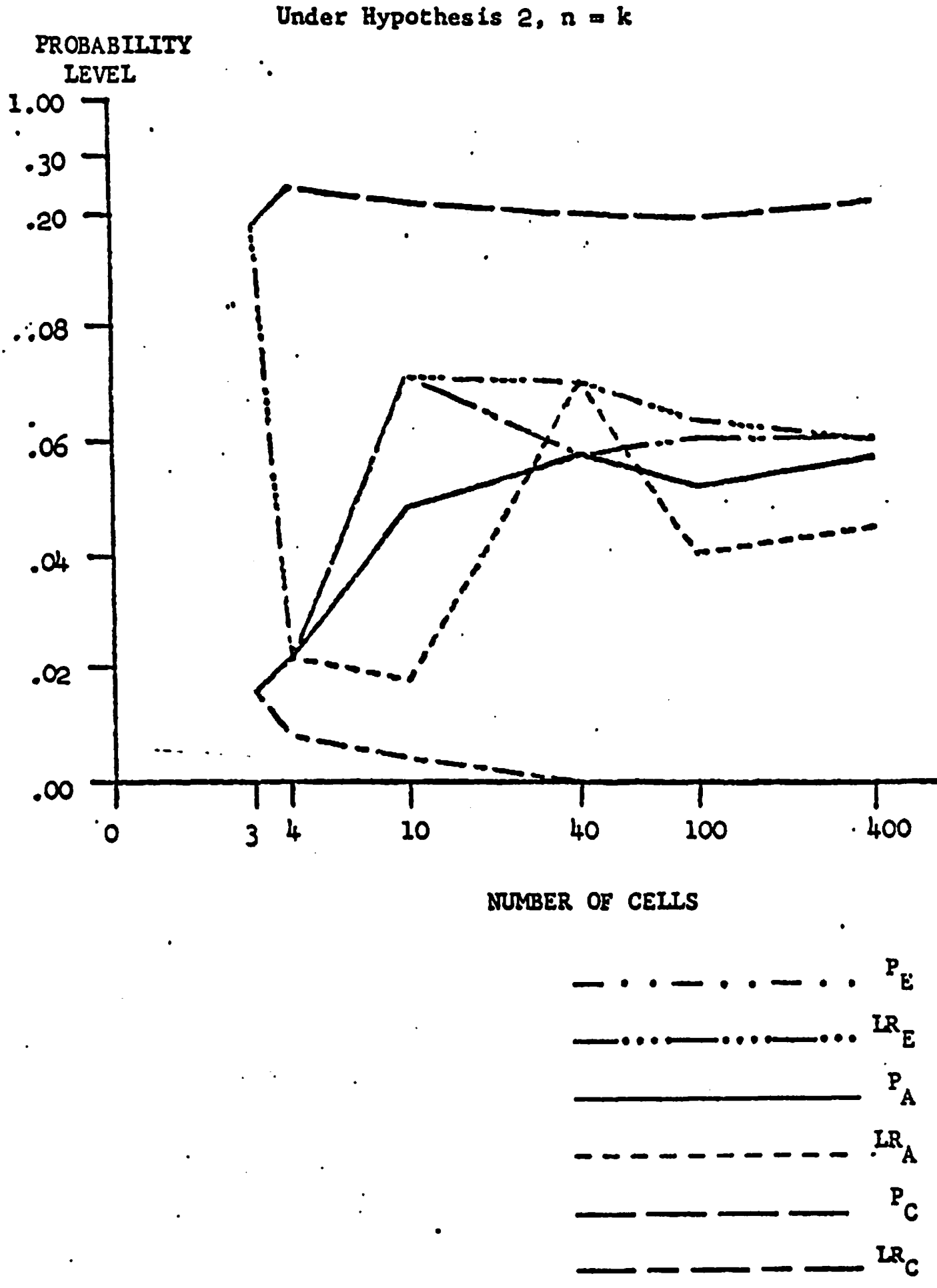


FIGURE K

Estimated Probability of Exceeding ${ }^{2} .99=2.326$

- Under Hypothes 1s 2 when $n * k$

\section{PROBABILITY}

:

IEVEI

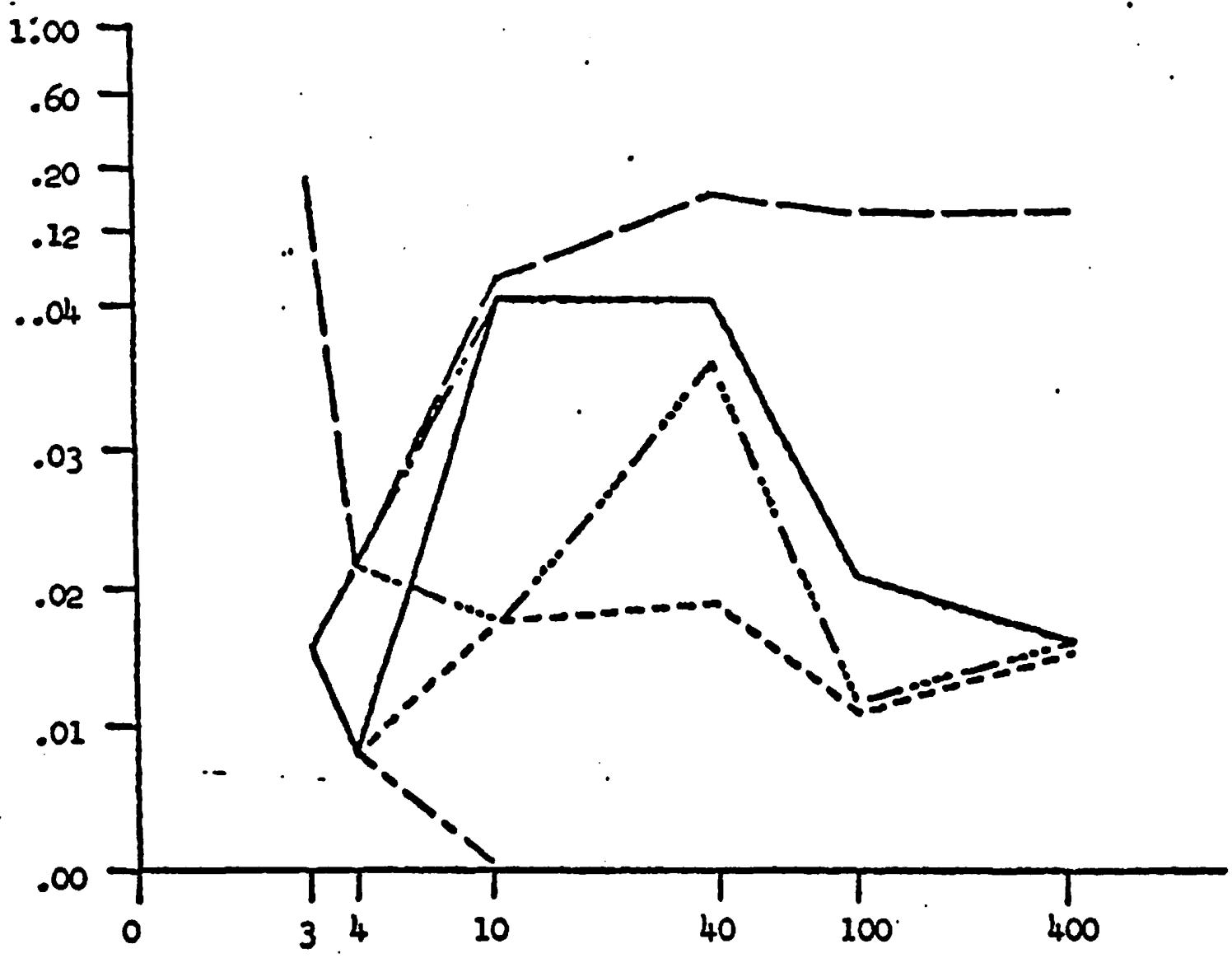

NUMBER OF CELIS

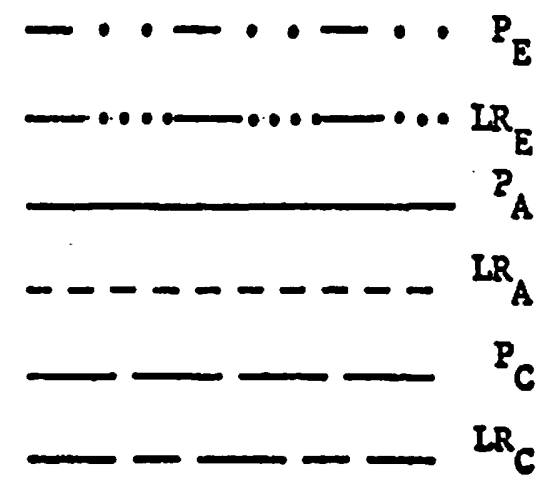


FIGURE I

$$
\text { Estimated Probability of Exceeding Z } .95=1.645
$$

Under Hypothesis 9 when $n=.5 k$

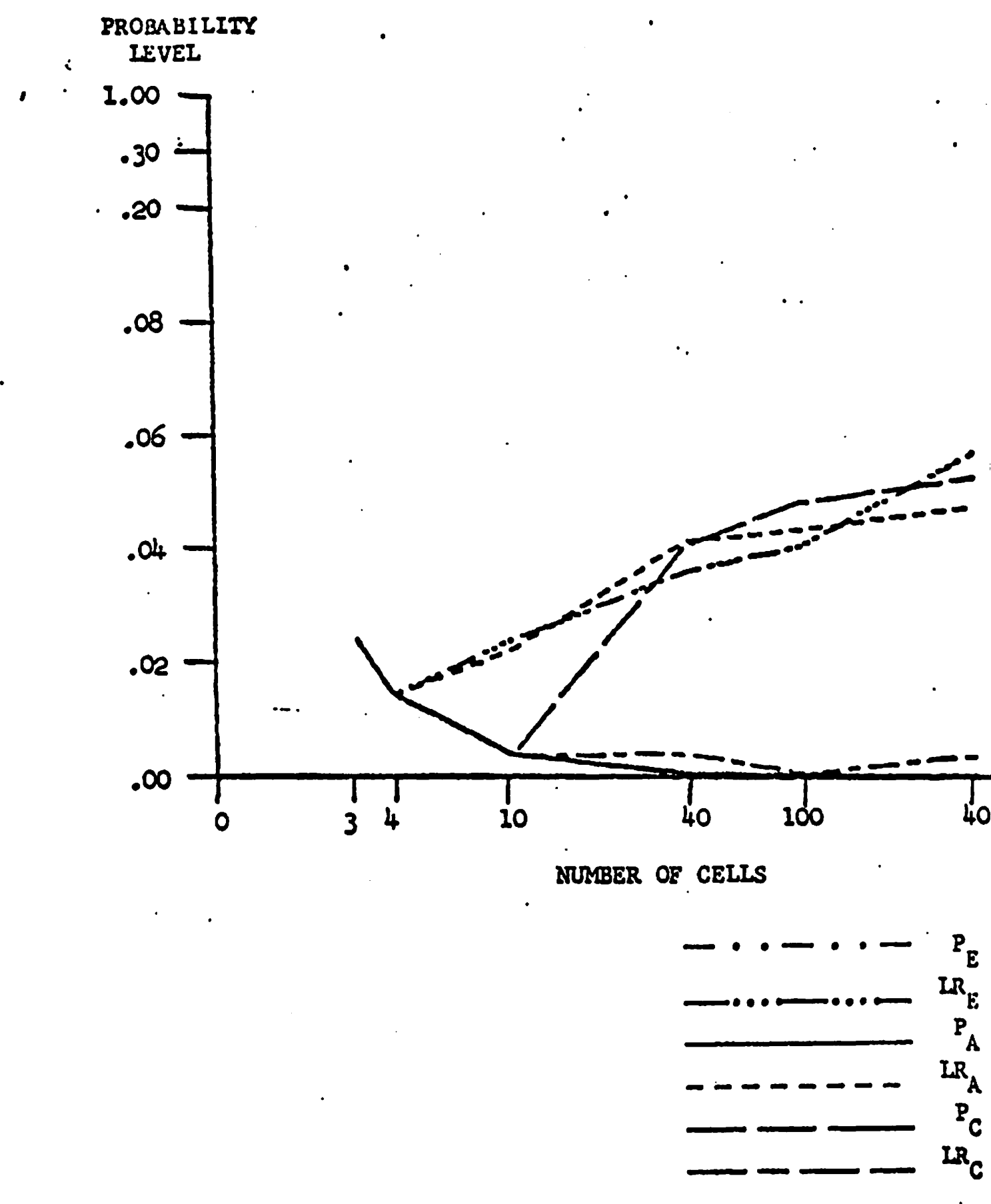




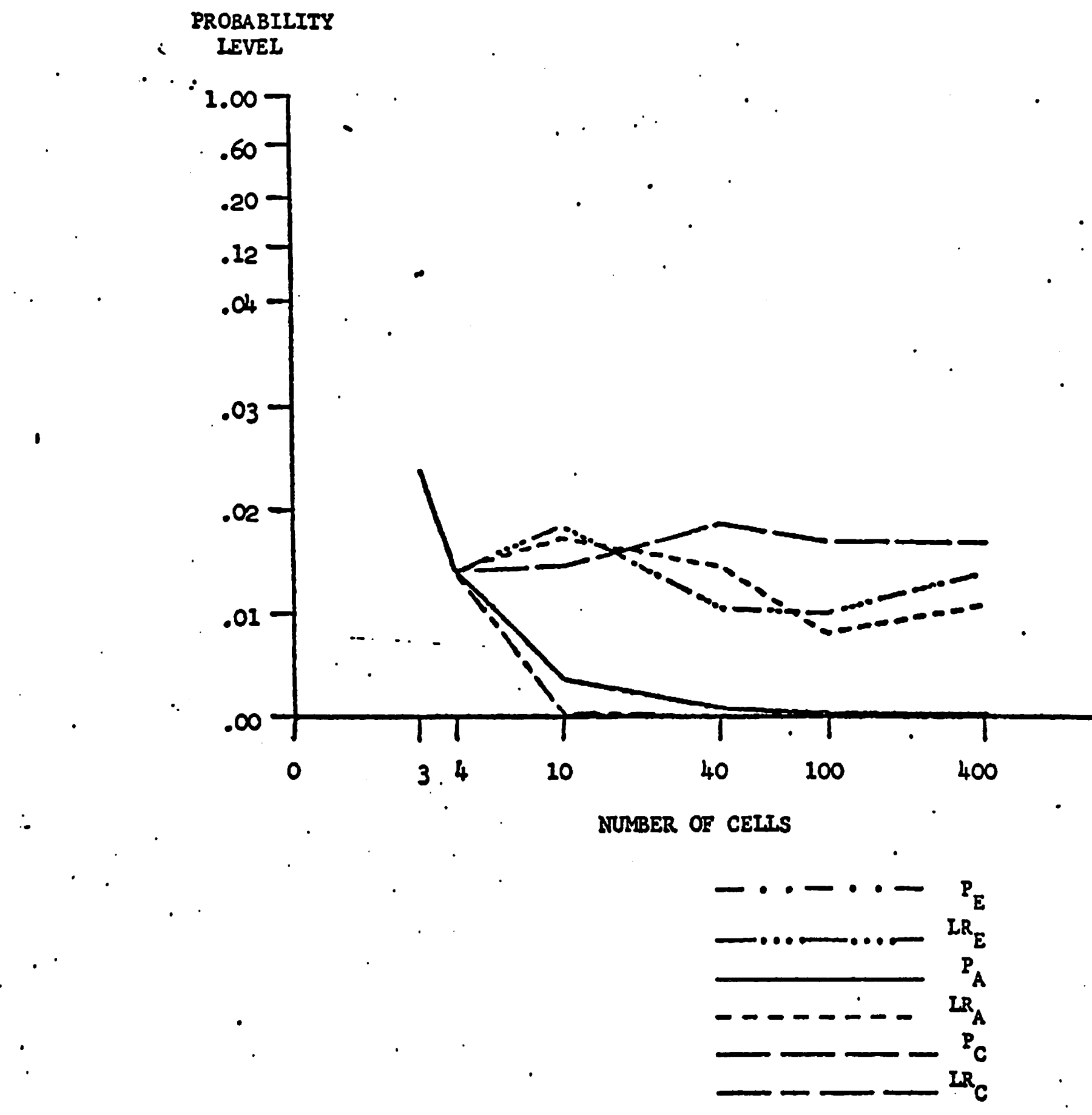

\section{FIGURE M \\ Estimated Probability of Exceeding $2.99=2.326$ \\ Under Hypothesis 9 when $n=. .5 k$}


Table 1

MINIMUM CONTRIBUTIONS FOR OBSERVED COUNTS OF 2ERO AND ONE

\begin{tabular}{|c|c|c|c|c|c|c|}
\hline $\begin{array}{l}\text { Expected } \\
\text { Frequency }\end{array}$ & $\frac{\text { Count }}{x^{2}}$ & $\frac{\text { f Zero }}{G^{2}}$ & $\begin{array}{c}\text { Prob } \\
\text { (Zero Count) } \\
\text { Under } \\
\text { Poisson }\end{array}$ & $\frac{\text { Count }}{x^{2}}$ & $\frac{\text { of One }}{G^{2}}$ & $\begin{array}{c}\text { Prob } \\
\text { (One Count) } \\
\text { Under } \\
\text { Polsson }\end{array}$ \\
\hline 5.00 & 5.00 & 10.00 & .00674 & 3.200 & 4.781 & .03369 \\
\hline 3.00 & 3.00 & 6.00 & .04979 & 1.333 & 1.803 & .14936 \\
\hline 2.00 & 2.00 & 4.00 & .13533 & 0.500 & 0.614 & .27067 \\
\hline 1.50 & 1.50 & 3.00 & .22313 & 0.167 & 0.189 & .33470 \\
\hline 1.00 & 1.00 & 2.00 & .36788 & 0.000 & 0.000 & .36788 \\
\hline 0.75 & 0.75 & 1.50 & .47237 & 0.083 & 0.074 & .35427 \\
\hline 0.50 & 0.50 & 1.00 & .60653 & 0.500 & 0.386 & .30326 \\
\hline 0.25 & 0.25 & 0.50 & .77880 & 2.250 & 1.273 & .19470 \\
\hline 0.10 & 0.10 & 0.20 & .90483 & 8.100 & 2.806 & .09048 \\
\hline 0.05 & 0.05 & 0.10 & .95123 & 18.050 & 4.091 & .04756 \\
\hline 0.01 & 0.01 & 0.02 & .99004 & 98.010 & 7.230 & .00990 \\
\hline
\end{tabular}

NOTE: Nirime contribution for $G^{2}$ is $\lim _{n \rightarrow \infty} 2 n \log \left(n /\left(n-n p_{j}\right)\right)=2 n p_{j}$ for $2 z=00$ count in the $j-$ th cell, and $11 m_{n \rightarrow \infty} 2 \log \left(1 / n p_{j}\right)+2(n-1) \log \left((n-1) /\left(n-n p_{j}\right)\right)$ $=-i \operatorname{iog}\left(n p_{j}\right)+2\left(n p_{j}-1\right)$ for a count of one. It is interesting to note the values for $G^{2}$ are limits of the OUTLIER values given by Gokhale and Kullback (1978, p. 64). 
Table 2

MULI EYPOITIESES CONSIDERED IN THE STUDY

Label

1

2

3

4

5

6

7

8

9
NuIl Hypotheses

$$
\begin{aligned}
& \left(\frac{1}{k}, \frac{1}{k}, \ldots, \frac{1}{k}\right) \\
& .1\left(\frac{1}{k}, \frac{1}{k}, \ldots, \frac{1}{k}\right)+.9(1,0, \ldots, 0) \\
& \left(\frac{3}{8}+\frac{1}{2 k}, \frac{1}{8}+\frac{1}{2 k}, \frac{1}{2 k}, \ldots, \frac{1}{2 k}\right) \\
& .9\left(\frac{1}{k}, \frac{1}{k}, \ldots, \frac{1}{k}\right)+.1\left(\frac{1}{2}, \frac{1}{2}, 0, \ldots, 0\right)
\end{aligned}
$$$$
\left(c_{1}, c_{2}, \ldots, c_{k}\right) \text {, where } c_{1}=\frac{\dot{1}}{k} \sum_{j=1}^{k} \frac{1}{j}
$$$$
.1\left(c_{1}, c_{2}, \ldots, c_{k}\right)+.9\left(\frac{1}{k}, \frac{1}{k}, \ldots, \frac{1}{k}\right)
$$$$
.1\left(c_{1}, c_{2}, \ldots, c_{k}\right)+.9(1,0, \ldots, 0)
$$$$
.1\left(c_{1}, c_{2}, \ldots, c_{k}\right)+.9\left(\frac{1}{2 k}, \frac{1}{2 k}, \ldots,\right.
$$

$$
\left.\frac{1}{2 k}, 0, \ldots, 0\right)
$$

$.1\left(c_{1}, c_{2}, \ldots, c_{k}\right)+.9\left(\frac{1}{k-1}, \ldots, \frac{1}{k-1}, 0\right)$ 
REFERENCES

Cochran, W.G. (1942), "The $\chi^{2}$ correction for continuity," Iowa State College Journal of Science, 16, 421-436.

Cochran, W.G. (1954), "Some methods for strengthening the common $X^{2}$ tests," Blometrics, 10, 417-451.

Gokhale, D.V. and Solomon Kullback (1978), The Information in

Contingency Tables. New York, Marcel Dekker, Inc.

Good, I.J., I.N. Grover, and G.J. Mitchell (1970), "Exact distributions for $\mathrm{x}^{2}$ and for the likelihood-ratio statistic for the equiprobable multinomial distribution," Journal of the American Statistical Association, 65, 267-283.

Haldane, J.B.S. (1937), "The exact value of moments of the distribution of $X^{2}$, used as a test of goodness of fit when expectations are small," Biometrika, 29, 133-143.

Hoeffding, W. (1965), "Asymptotically optimal tests for multinomial distributions," Annals of Mathematical Statistics, 36, 369-401.

Holst, L. (1972), "Asymptotic normality and efficiency for certain goodnessof-fit tests," Biometrika, 59, 137-145.

Holst, L. (1976), "On multinomial sums," Mathematics Research Center Technical Summary Report No. 1629, University of Wisconsin-Madison.

Katt1, S.J. (1973), "Exact distribution for the ch1-square test in the one way table," Communications in Statistics, 2 , 435-447.

Koehler, K.J. (1977), Goodness-of-fit statistics for large sparse mult1nomials, Unpublished Ph.D. dissertation, School of Statistics, University of Minnesota.

Larntz, K. (1978), "Small-sample comparisons of exact levels for chi-squared goodness-of-fit statistics," Journal of the American Statistical Association, 73, 253-263.

Morr1s, C. (1966), "Admissable Bayes procedures and classes of epsilon Bayes procedures for testing hypotheses in a multinomial distribution," Technical Report No. 55, Department of Statistics, Stanford University.

Morris, C. (1975), "Central limit theorems for multinomial sums," Annals of Statistics, $\underline{3}, 165-188$. 
Nass, C.A.G. (1959), "The $\chi^{2}$ test for small expectations in contingency tables, with special reference to accldents and absenteeism," Biometrika, 46, 365-385.

Neyman, J. and E.S. Pearson (1928), "On the use and Interpretation of certain test criterla for purposes of statistical iriference," Biometrika, 20-A, 175-247, 264-299.

Pearson, K. (1900), "On the criterion that a given system of deviations from the probable in the case of a correlated system of variables is such that it can be reasonably supposed to have arisen from random sampling," Phil. Mag., 50, 157-175.

Roscoe, J.T. and J.A. Byars (1971), "An investigation of the restraints with respect to the sample size commonly imposed on the use of the chi-square statistics," Journal of the American Statistical Association, 66, 755-759.

Slakter, M.J. (1966), "Comparative validity of the chl-square and two modifled ch1-square goodness-of-fit tests for small but equal expected frequencies," Biometrika, 53, 619-622.

Steck, G.P. (1957), "Limit theorems for conditional distributions," University of Califomia Publications in Statistics, Vol. 2, No. 12, 237-284.

Vessereau, A. (1958), "Sur les conditions d'application du criterium $X^{2}$ de poisson," Bulletin International Institute of Statistics, 36 , 87-101.

West, E.N. and 0. Kempthorne (1971), "A comparison of the $\mathrm{chl}^{2}$ and likelihood ratio tests for composite alternatives," Journal of Statistical Computation and Simulation, 1, 1-33.

Zahn, P.A. and G.C. Roberts (1971), "Exact $X^{2}$ criterion tables with cell expectation one: An application to Coleman's measure of consenses," Journal of the American Statistical Association, 66, 145-148. 